\title{
The Columbia University Scale for Psychopathology in Alzheimer's Disease
}

\author{
D. P. Devanand, MD; Lisa Miller; Marcus Richards, PhD; Karen Marder, MD, MPH; Karen Bell, MD; \\ Richard Mayeux, MD; Yaakov Stern, PhD
}

- The Columbia University Scale for Psychopathology in Alzheimer's disease is a new screening instrument developed for use by clinicians or trained lay interviewers. Interrater reliability was established between a psychiatrist and a lay interviewer in $\mathbf{2 0}$ patients. In an independent sample of 91 outpatients with very mild to moderate probable Alzheimer's disease, caregiver informants reported that depressed mood was common $(46.2 \%)$ but rarely persistent $(2.2 \%)$, and that sleep disturbance occurred frequently $(41.8 \%)$ but was never severe $(0 \%)$. There were significant but weak associations between the presence of specific subtypes of delusions and severity of dementia. Although a variety of delusional symptoms were reported, they were frequently transient and patients often accepted the truth if corrected by the caregiver. As a result, few patients met broad or narrow operational criteria used to define delusions. Prior studies may have overestimated the prevalence of psychotic features in Alzheimer's disease by not employing standard definitional criteria. The findings also indicate that new methodology such as that employed in this instrument needs to be evaluated more widely.

(Arch Neurol. 1992;49:371-376)

D syc sychosis, behavioral disturbances, and depressive symptoms occur frequently in patients with Alzheimer's disease (AD), ${ }^{1,2}$ are distressing to patients and caregivers, 3,4 are often treated with psychotropic medications, ${ }^{2,5}$ and may be associated with the likelihood of institutionalization. ${ }^{6}$ Recent instruments that have been developed to evaluate psychopathology in AD rely on the use of trained clinician interviewers. ${ }^{2,7}$ We developed the Columbia University Scale for Psychopathology in Alzheimer's Disease (CUSPAD), a short semi-structured instrument that can be administered by a trained lay interviewer for use in cross-sectional and long-term follow-up studies. Particular emphasis was placed on the use of specific follow-up questions to help establish operational definitions for the presence of psychotic features in AD, since this has been lacking in prior instruments of this type. We

\section{Accepted for publication October 21, 1991.}

From the Memory Disorders Clinic in the New York State Psychiatric Institute, and the Center for Alzheimer's Disease Research in New York City (Drs Devanand, Richards, Marder, Bell, Mayeux, Stern, and Ms Miller) and the Departments of Psychiatry (Dr Devanand) and Neurology (Ms Miller and Drs Richards, Marder, Bell, Mayeux, and Stern), College of Physicians and Surgeons of Columbia University, New York, NY.

Reprint requests to the New York State Psychiatric Institute, 722 W 168th St, New York, NY 10032 (Dr Devanand). describe interrater reliability between a lay interviewer (L.M.) and a research psychiatrist (D.P.D.), divergent validity, prevalence of symptoms, and relations between scale items and severity of dementia as assessed by cognitive function and ability to perform activities of daily life.

\section{SUBJECTS AND METHOD Scale Development and Design}

A few items were adapted from existing instruments ${ }^{2}$ and other items were developed de novo by the principal developer (D.P.D.) and other research personnel (Y.S. and M.R.). An earlier version of the scale was piloted before the current version was finalized. The scale takes approximately 10 to 25 minutes to administer to an informant, and focuses on symptoms during the past month. There is a single explicit question for each item to facilitate its use by lay interviewers.

Operational definitions for delusions, hallucinations, and illusions were based on standard definitional criteria for these symptoms. ${ }^{8}$ The informant's report of the patient's behavioral response, if any, is taken into account to score psychotic symptoms. Psychotic symptoms in Alzheimer's disease are known to be different from those seen in other psychotic disorders. ${ }^{2,9}$ More critically, delusions are frequently transient, and patients with $\mathrm{AD}$ will often accept the truth if corrected by the caregiver, even though the same symptom may recur shortly thereafter as a result of amnesia. These features can make it difficult to ascertain the presence of a "true" delusion fulfilling traditional definitional criteria. ${ }^{8}$ Hence, in the CUSPAD, the initial question for each type of delusion is scored dichotomously (present/absent). If a delusional symptom is present, two additional questions are asked. The first follow-up question inquires if the delusion is persistent (more than three times per week) or transient, and the second question inquires if the patient accepts correction of the false belief from the caregiver. A "broad" definition of a delusion is met when the patient does not accept the caregiver's correction of the false belief/idea, while a "narrow" definition of a delusion is met when the patient does not accept the caregiver's correction of the false belief/idea and the symptom is persistent, ie, occurs more than three times per week.

Hallucinations, if present, are rated as "vague" or "clear." This approach to rate hallucinations was adopted a priori because this interview relies solely on the informant's report, and patients are known to manifest behaviors that are often difficult to interpret clearly as hallucinations. Illusions are rated similarly to hallucinations.

Wandering, verbal outbursts, violence, agitation, and sundowning constitute the items for behavioral disturbance that are scored as present or absent. Items for depression include depressed mood, difficulty sleeping and change in appetite, and are scored on a 5-point scale to ascertain frequency and severity of these symptoms. Since the interview was developed primarily as a screening instrument, the addition of more items was not entertained. 


\begin{tabular}{|c|c|c|}
\hline Table 1.- Interrater & $\begin{array}{l}\text { sility for } \mathrm{Pr} \\
\text { iptoms }\end{array}$ & ce/Absence \\
\hline \multirow[b]{2}{*}{ Symptom Category } & \multicolumn{2}{|c|}{ Kappa Coefficient } \\
\hline & $\begin{array}{c}\text { Conjoint } \\
\text { Interviews }\end{array}$ & $\begin{array}{l}\text { Independent } \\
\text { Interviews }\end{array}$ \\
\hline \multicolumn{3}{|l|}{ Delusions } \\
\hline Present/absent & .77 & .61 \\
\hline Persistent/transient & .90 & .30 \\
\hline Accepts truth & .74 & .54 \\
\hline Hallucinations & 1.0 & .63 \\
\hline Illusions & 1.0 & .62 \\
\hline Behavioral disturbance & .88 & .67 \\
\hline Depression & .80 & .73 \\
\hline
\end{tabular}

\section{Subjects}

Subjects were outpatients attending a memory disorders clinic, and met the Diagnostic and Statistical Manual of Mental Disorders, Revised Third Edition criteria for primary degenerative dementia of the Alzheimer type and National Institute of Neurological Disorders and Stroke-Alzheimer's Disease and Related Disorders Association criteria for probable AD. ${ }^{10}$ Exclusion criteria were alcohol or drug dependence during the 5 years prior to the first clinic visit, primary affective disorder within 1 year prior to onset of dementia, and history or clinical evidence of other causes of dementia excluded by differential diagnostic work-up including detailed neurologic and psychiatric evaluation, computed tomographic scan of the brain (or magnetic resonance imaging if clinically indicated), electroencephalogram, relevant blood studies, and cerebrospinal fluid studies if indicated. Reliability was assessed by interviewing informants from a group of 20 patients with $\mathrm{AD}$ at all stages of severity. As part of a baseline evaluation in a longitudinal follow-up study, the trained lay interviewer conducted informant interviews in an independent sample of $91 \mathrm{AD}$ patients with mild to moderate dementia (Clinical Dementia Rating of 1 or 2). ${ }^{11}$ All 91 patients were free of psychotropic medication at the time of evaluation.

\section{RESULTS \\ Reliability}

The scale was administered to an informant. After a brief piloting phase in seven patients to establish initial agreement on rating criteria, interrater reliability was evaluated between a trained lay interviewer (L.M.) and a research psychiatrist (D.P.D.) experienced in the evaluation of patients with AD. First, the lay interviewer conducted 10 interviews with the psychiatrist present in the room, and the two raters subsequently completed scoring for the scale independently. Second, the lay interviewer and the psychiatrist conducted 10 back-to-back independent interviews in an independent sample. Rates of agreement were calculated by the kappa coefficient, which measures the ratio of the observed agreement beyond chance to the potential agreement beyond chance, with a kappa of 1.0 representing complete agreement beyond chance and a kappa of 0 indicating agreement at the chance level. Interrater reliability was high for independent ratings from conjoint interviews (kappa's ranging from .74 to 1.0 , Table 1 ). Reliability was also high for the independent interviews that were conducted back-to-back with the same informant (kappa's ranging from .54 to .73), with the exception of the rating for a delusion being persistent or transient where the two raters agreed in seven of 10 cases (kappa $=.30$ ).
Table 2.-Demographic Characteristics of the Sample (91 Outpatients With AD)*

\begin{tabular}{|lrccc|}
\hline \multicolumn{1}{|c}{ Demographics } & Mean & SD & Minimum & Maximum \\
\hline Sex, \% female & 56 & & & \\
Age, $y$ & 70.8 & 8.3 & 49 & 86 \\
Age at onset, $y$ & 67.2 & 8.6 & 46 & 84 \\
Duration of illness, $y$ & 3.5 & 1.8 & 1 & 9 \\
Presenile onset, \% & $29 \%$ & & & \\
Education, $y$ & 13.3 & 3.7 & 1 & 20 \\
mMMSE & 36.3 & 6.5 & 23 & 51 \\
BDRS & 3.8 & 1.9 & 0.5 & 12 \\
\hline
\end{tabular}

*AD indicates Alzheimer's disease; mMMSE, modified MiniMental State Examination, maximum score 57; and BDRS, Blessed Dementia Rating Scale part 1 (functional activity component only).

\section{Patient Demographics}

The mean age was 72.09 years (SD, 9.77), 29\% of patients had their onset of illness before age 65 years, and $65 \%$ were women (Table 2). Patients with presenile onset did not differ from those with senile onset on measures of cognitive function (modified Mini-Mental State Examination [mMMSE], maximum score, 57$)^{12}$ or on ability to perform activities of daily life (Blessed Dementia Rating Scale part 1 [BDRS]), ${ }^{13}$ although it must be emphasized that the range of severity of dementia was restricted a priori in this sample.

\section{Divergent Validity}

For the 91 subjects, scores for presence (score $=1$ ) or absence (score $=0$ ) of relevant items were summed to obtain several continuous measures: paranoid delusions, misidentification delusions, all delusions combined, hallucinations, all items for psychosis, behavioral disturbance, depression, and the sum of all scale items. Neither behavioral disturbance nor depression correlated significantly with any subset of items for delusions (Table 3), and there was a low correlation between paranoid delusions and delusions of misidentification $(r=.12)$. The weak correlations observed between item subsets suggest that they measure different entities that do not share much common variance.

\section{Prevalence of Delusional Symptoms}

Fifty percent of informants (27/54) who initially reported that the patient had no strange ideas subsequently responded positively to questions inquiring about specific delusions. The most common delusional symptoms reported as present were the idea that others were stealing from the patient (18.7\%) and misidentification (12.1\%). The least common delusional symptoms were the belief that the spouse was unfaithful $(1.5 \%)$, and the belief that the patient's image in a mirror was someone else $(1.1 \%)$. There was a striking drop-off in prevalence for all delusional items when positive responses were followed up with questions to assess if the patient met criteria for a broad definition or narrow definition of a delusion (Table 4). While $18.7 \%$ of patients had indicated to their informants that they believed someone was stealing from them, only $13.2 \%$ and $4.4 \%$ met the broad and narrow definitions of a delusion in this category, respectively. For 


\begin{tabular}{|c|c|c|}
\hline $\begin{array}{l}\text { Table 3.-Correlations Betw } \\
\text { for Psychotic Features (Pres } \\
\text { Behavioral Disturbances or }\end{array}$ & $\begin{array}{l}\text { een Subsets } \\
\text { nv } / \text { Absent } O \\
\text { repressive } \mathbf{S}\end{array}$ & $\begin{array}{l}\text { of ltems } \\
\text { nly) and } \\
\text { imptoms }\end{array}$ \\
\hline \multirow[b]{2}{*}{$\begin{array}{c}\text { Presence of Psychotic Features, } \\
\text { Sum of Item Scores }\end{array}$} & \multicolumn{2}{|c|}{$\begin{array}{c}\text { Correlation Coefficient } \\
\text { (Pearson's } r \text { ) }\end{array}$} \\
\hline & $\begin{array}{l}\text { Behavioral } \\
\text { Disturbance }\end{array}$ & Depression \\
\hline Paranoid delusions & .19 & -.09 \\
\hline Misidentification & .11 & .21 \\
\hline Sum score of all delusions & .20 & .12 \\
\hline Hallucinations & .07 & .13 \\
\hline Sum score of all psychotic items & .20 & .15 \\
\hline
\end{tabular}

\begin{tabular}{|c|c|c|c|}
\hline \multirow[b]{3}{*}{ Delusional Symptoms } & \multirow{3}{*}{$\begin{array}{c}\text { Present, } \\
\% \\
\text { Total } \\
\text { Sample }\end{array}$} & \multirow{2}{*}{\multicolumn{2}{|c|}{$\begin{array}{l}\text { "True Delusion," } \\
\% \text { Total Sample }\end{array}$}} \\
\hline & & & \\
\hline & & $\begin{array}{c}\text { Broad } \\
\text { Definition }\end{array}$ & $\begin{array}{c}\text { Narrow } \\
\text { Definition }\end{array}$ \\
\hline Strange ideas, general question & 16.5 & 14.3 & 4.4 \\
\hline Stealing from patient & 18.7 & 13.2 & 5.5 \\
\hline Spouse unfaithful & 1.5 & 1.1 & 0 \\
\hline Other unfounded suspicions & 7.7 & 6.6 & 2.2 \\
\hline Suspects abandonment & 4.4 & 1.1 & 0 \\
\hline Somatic delusions & 6.6 & 3.3 & 3.3 \\
\hline Stated someone home & 6.6 & 5.5 & 2.2 \\
\hline Image in mirror is not patient & 1.1 & 1.1 & 0 \\
\hline Misidentified people & 12.1 & 4.4 & 1.1 \\
\hline Thinks own house is not home & 5.5 & 2.2 & 0 \\
\hline Television characters are real & 3.3 & 1.1 & 1.1 \\
\hline Other strange ideas & 3.3 & 0 & 0 \\
\hline
\end{tabular}

*AD indicates Alzheimer's disease. Broad definition of a true delusion: the patient does not accept the caregiver's correction of the false belief/idea, and the symptom may be persistent or transient. Narrow definition of a true delusion: the patient does not accept the caregiver's correction of the false belief/idea, and the symptom is persistent, ie, occurs at least three times per week.

the other common symptom of a misidentification delusion, the prevalence dropped from $12.1 \%$ to $4.4 \%$ (broad definition) and $1.1 \%$ (narrow definition), respectively. With the broad definition of a delusion, only one symptom occurred in more than $10 \%$ of the sample, namely, that someone was stealing from the patient. For the narrow definition of a delusion, the prevalence rates were strikingly lower (Table 4).

\section{Other Symptoms}

Hallucinations, rated as "vague" or "clear," were rarely reported. Auditory hallucinations occurred in $1.1 \%$ of patients, visual hallucinations in $4.4 \%$, olfactory hallucinations in $3.3 \%$, and tactile hallucinations in $1.1 \%$ (Table 5). Agitation was the most common behavioral disturbance $(46.2 \%)$, although it was reported as mild by most informants. Depressed mood (46.2\%) and sleep disturbance $(41.8 \%)$ were also commonly reported, but few patients had depressed mood "all the time" $(2.2 \%)$ and none

\begin{tabular}{|c|c|}
\hline \multicolumn{2}{|c|}{$\begin{array}{l}\text { Table 5.-Prevalence of Symptoms Other } \\
\text { Than Delusions in } 91 \text { Alzheimer's Disease Patients } \\
\text { With Mild to Moderate Dementia }\end{array}$} \\
\hline Other Symptoms & $\begin{array}{c}\text { Prevalence, } \\
\%\end{array}$ \\
\hline \multicolumn{2}{|l|}{ Hallucinations } \\
\hline Auditory & 1.1 \\
\hline Visual & 4.4 \\
\hline Olfactory & 3.3 \\
\hline Tactile & 1.1 \\
\hline Wandering & 5.5 \\
\hline Verbal outbursts & 20.9 \\
\hline Physical threats & 5.5 \\
\hline Agitation & 46.2 \\
\hline More confused at night & 17.6 \\
\hline Depressed mood & 46.2 \\
\hline Occasionally & 25.3 \\
\hline Some of the time & 17.6 \\
\hline Most of the time & 8.8 \\
\hline All the time & 2.2 \\
\hline Sleep difficulty & 41.8 \\
\hline Slight & 15.4 \\
\hline $\begin{array}{l}\text { More than } 2 \text { hours sleep } \\
\text { per night }\end{array}$ & 15.4 \\
\hline $\begin{array}{l}\text { Less than } 2 \text { hours sleep } \\
\text { per night }\end{array}$ & 0 \\
\hline Excessive sleep & 11.0 \\
\hline Change in appetite & 34.1 \\
\hline Slight & 11.0 \\
\hline No appetite & 4.4 \\
\hline Needs persuasion to eat & 5.5 \\
\hline Excessive appetite & 13.2 \\
\hline
\end{tabular}

had severe sleep disturbance with less than 2 hours sleep per night $(0 \%$, Table 5$)$.

\section{Relations to Demographic Variables}

Women scored higher on the summed score for the "presence" of paranoid delusions $(t=3.1, P=.003)$ and the sum score for all delusions $(t=2.2, P<.05)$, but did not differ from men on cognitive performance (mMMSE) or functional activity (BDRS). Age showed a weak association with the presence of paranoid delusions $(r=.21$, $P<.05)$, and correlated inversely with the presence of hallucinations $(r=-.32, P=.002)$. Age at onset showed a strong inverse correlation with the presence of hallucinations $(r=-.35, P<.001)$, but with none of the other measures. There were no significant correlations between duration of illness and any of the measures examined. Education was inversely related to the presence of paranoid delusions $(r=-0.27, P<.01)$ and the sum of all items for psychosis $(r=-.21, P<.05)$, but not to any of the other summed measures.

\section{Relations to Cognition and Functional Impairment}

Cognitive impairment as assessed by mMMSE scores showed significant inverse correlations with the presence 
of delusions of misidentification $(r=-.30, P=.004)$, sum of all items for delusions $(r=-.30, P=.004)$, the sum of all items for psychosis $(r-.32, P=.002)$, depressive symptoms $(r=-.27, P<.01)$, and sum total psychopathology scores $(r=-.37, P<.001)$, but did not correlate significantly with paranoid delusions, hallucinations, or behavioral disturbance. Increasing cognitive impairment was associated with greater psychopathology. When the broad definition of a delusion was used to obtain summed scores for item subsets, all the associations held except for the relations between delusions of misidentification and mMMSE scores that were no longer significant. When the narrow definition of a delusion was used similarly, the only significant association obtained was an inverse relationship between mMMSE scores and sum total psychopathology scores $(r=-.28, P<.01)$.

The sample was dichotomized into separate groups with very mild dementia ( $\mathrm{mMMSE} \geqslant 40, \mathrm{n}=31$ ) and mild to moderate dementia (mMMSE $\leqslant 39, n=60$ ). Using the summed scores from responses to the presence/absence items, patients with mMMSE scores of 39 or less reported more delusions of misidentification $(t=2.1, P<.05)$ and more delusions overall $(t=2.4, P<.05)$, as well as more depression $(t=2.3, P<.05)$ than those with mMMSE scores of 40 or more. These associations with delusions of misidentification and delusions overall disappeared when the broad or narrow definitions for delusions were used to calculate the summed scores, with the exception of the sum total psychopathology score that was associated with greater cognitive impairment (broad definition, $t=2.24, P<.05$; narrow definition, $t=2.27, P<.05$ ).

Functional activity level as assessed by BDRS part 1 scores (excluding behavioral and cognition items) were associated with the summed psychopathology scores when the presence/absence scoring for delusions was used $(r=.30, P<.005)$, in the direction of increased psychopathology being associated with increased functional impairment. The BDRS scores correlated significantly with the presence of delusions of misidentification $(r=.28, P<.01)$ and depressive symptoms $(r=.24$, $P=.02$ ), but not with any of the other summed scores for subsets of items for psychosis or behavioral disturbance. However, when delusions were restricted to the broad or narrow definitions, there were no significant correlations between BDRS scores and the item subsets for delusions and psychosis.

\section{COMMENT}

The CUSPAD is a new instrument that is quick and easy to use, yet provides valuable information about the psychopathology of the patient with $\mathrm{AD}$. The good interrater reliability established between a lay interviewer (L.M.) and a research psychiatrist (D.P.D.) suggests that this scale is appropriate for use by trained lay interviewers. Informants frequently reported that the patient had no strange ideas, but subsequently answered positively to questions regarding specific delusions, suggesting that specific questions regarding delusional items are necessary to elicit symptoms. As described, for items pertaining to delusions, the new approach adopted proved to be valuable. First, it helped clarify the extent of "delusionality" for the lay interviewer, who indicated she might have otherwise have found it difficult to make this judgment in several instances where the initial presence/absence question was met by an ambiguous response from the infor- mant. Second, there was a striking drop-off in prevalence for all delusional items when the two follow-up questions were used directly to define a delusion as broad or narrow (Table 4). In addition, the associations between measures of psychosis and both cognitive function (mMMSE) and functional impairment (BDRS) decreased when the broad or narrow definition of a delusion was used to compute item subset scores. This might be due to the smaller number of subjects who met these criteria for delusions and/or the restriction of range of severity of dementia in this sample. Nevertheless, these findings raise issues that merit further discussion.

It is well known that the psychotic features exhibited by patients with $A D$ are different from those seen in other psychoses such as schizophrenia, and that the presence of amnesia in AD may result in a situation where the patient will accept the truth when corrected yet bring up the same delusional complaint at a later time. ${ }^{2,9}$ In addition, amnesia and related cognitive impairments may impact on the frequency with which a symptom is reported by the patient. These factors were taken into account in the design of the CUSPAD. The decline in prevalence rates with the broad and narrow definitions for delusions suggests that prior studies that did not clarify exactly how a "delusion" was defined may have overestimated the prevalence of delusions by including those symptoms that were of ideational rather than delusional intensity. A recent study of patients with $\mathrm{AD}$ found that persecutory ideas not held with delusional intensity were more common than delusions of theft and suspicion, ${ }^{14}$ supporting the notion that the wide variability noted in the prevalence of delusions in previous studies may have been due to problems in the definitional criteria employed as well as differences in sample characteristics. ${ }^{2,14,15}$ The presence of various psychotic and behavioral symptoms, as assessed by a positive score for the initial "presence/ absence" question, was not dissimilar to that reported in other recent studies, ${ }^{14,16,17}$ but prevalence rates for delusions using the broad and narrow definitions in the CUSPAD were lower. One limitation of direct comparisons across studies is the variation in ranges of dementia severity in different samples. With respect to directions for the future, studies should consider operationalizing assessment of delusions more strictly, as was done with the CUSPAD.

For hallucinations, the terminology vague and clear made it easy to classify the responses obtained, and facilitated scoring by the lay interviewer. One broad caveat that limits the use of the phrases "true delusion" or "clear hallucination" in this context is that the source of all the information in this study was the informant. However, this is often unavoidable in outpatients with AD who cannot be observed directly by clinicians for an extended period of time. ${ }^{2,14}$ The higher prevalence of both visual and auditory hallucinations reported in other studies s.18 $^{2,18}$ might be related to severely demented patients not being included in this sample.

The components that measured behavioral disturbance and depression did not correlate strongly with any of the psychotic syndromes evaluated with this scale, thus demonstrating divergent validity. Within this sample of only mild to moderate $\mathrm{AD}$, the associations between degree of psychopathology and severity of dementia as assessed by MMMSE and BDRS scores were not robust. There is some prior evidence supporting the notion that 
Delusions

For delusions, the answer to the initial question is rated: no, 0 or yes, 1 . If "yes", two follow-up questions are asked: (1) does this occur some of the time or most of the time?, with the answer rated as transient, 0 , or persistent, 1 , and $(2)$ will the patient accept the truth if corrected?, rated as no, 0 , or yes, 1

General question

In the past month, has he/she talked about any strange ideas or unusual beliefs?

Paranoid delusions

Has he/she suspected that his/her wife/husband is unfaithful?

Has he/she had any other unfounded suspicions?

Delusions of abandonment

Has he/she suspected or accused the caregiver of plotting to leave him/her?

Somatic delusions

Has he/she had any false beliefs that he/she has cancer or other physical illness?

Misidentification

Has he/she stated that people are in the home when nobody is there?

Has he/she looked in the mirror and said it was someone else?

Has he/she misidentified people, eg, said the spouse/caregiver is an imposter?

Has he/she said that his/her home is not his/her home?

Has he/she believed that the characters on television are real or in the room?

Other delusions

Has he/she had any false beliefs or other strange ideas that I have not asked?

Hallucinations

For hallucinations and illusions, answers are rated as no, 0 , or yes, 1. If "yes", a further rating is made as to whether the hallucination is vague, 1 , or clear, 2

Auditory: has he/she heard voices or sounds when no one is there?

Visual: has he/she seen visions?

Olfactory: Has he/she reported unusual smells like burning rubber or gas?

Tactile: has he/she felt that things are crawling on his/her skin?

Has he/she reported any other hallucinations?

Illusions

Has he/she reported that one thing is something else, eg, saying that a pillow looks like a person or that a light bulb looks like a fire starting?

Behavioral disturbances

Items are rated as no, 0 , or yes, 1 , except for the item for physical aggression that is rated as no, 0 ; threatening behavior, 1 ; physical violence, 2

Has he/she wandered away from home or the caregiver?

Has he/she made verbal outbursts?

Has he/she used physical threats and/or violence?

Has he/she been agitated or restless?

Has he/she become more confused in the evening/night compared with the day?

Depression

Ratings are described for each item below

Has he/she been sad, depressed, blue, or down in the dumps? No, 0; yes, 1

If "yes", is he/she depressed occasionally (score =1), some of the time (2), most of the time (3), all the time (4), or not applicable $(0)$.

Has he/she had difficulty sleeping? No, 0 ; yes, 1

If "yes", is there slight difficulty (1), at least 2 hours sleep per night (2), less than 2 hours sleep per night (3), or excessive sleepiness (4)

Has his/her appetite changed? No, 0 ; yes, 1

If "yes", is it slightly decreased (1), there is no appetite and food is tasteless (2), needs persuasion to eat at all (3), or excessive appetite (4)

*The time frame of interest is the past month. Presence of symptoms is rated in absolute fashion without reference to baseline symptom status, except for the items for sleep and appetite disturbance. Scoring is biased toward the positive, in that a single instance of a symptom yields a positive score. All questions are addressed to a caregiver informant. A detailed expanded version of the instrument is available from the authors on request. 
psychosis and behavioral disturbances are associated with increasing cognitive impairment. ${ }^{16,19}$ However, nonpsychotic behavioral disturbances did not show any relations to dementia severity, perhaps due to the restriction in range of severity of dementia in this sample.

Depressive symptoms increased with increasing cognitive impairment, in apparent contradiction to an earlier belief that depression tends to occur during the early stages of the illness. ${ }^{20,21}$ However, the findings are consistent with the study by Rubin and Kinscherf ${ }^{17}$ in which dysphoria occurred in $20 \%$ of patients with questionable dementia and in $27 \%$ of patients with mild dementia, and with a recent study that found depressed patients with $\mathrm{AD}$ to be more cognitively impaired and disabled than nondepressed patients. ${ }^{22}$ One possible explanation for these associations is that the prevalence of depression increases from the stage of questionable dementia up to the mild or moderately advanced stage before declining again with increasing severity of dementia at advanced stages of the illness.

The reported prevalence of depression in AD ranges from $0 \%$ to $50 \%$ in different studies, ${ }^{21-24}$ probably due to the application of different diagnostic methods and criteria. Family members report depression at far greater frequency than patients with $\mathrm{AD}$ report on direct interview. ${ }^{25,26}$ Also, symptoms such as loss of interest and pleasure are common to both depression and AD. ${ }^{27}$ The difficulty in ascertaining if such symptoms are part of a full depressive syndrome may partly underlie the difficulty in demonstrating efficacy for antidepressant medication in the treatment of depression in $\mathrm{AD},{ }^{28}$ although uncontrolled studies have been more optimistic. ${ }^{29,30} \mathrm{Al}-$ though depressed mood was reported in $46.2 \%$ of our sample, only $2.2 \%$ of patients were reported to be depressed all the time (Table 5). The findings suggest that a persistently depressed mood is uncommon in mild to moderate $\mathrm{AD}$, and the rarity of severe sleep disturbance reported provides indirect support. One limitation is that other features of the syndrome of depression were not systematically evaluated with the CUSPAD, a screening instrument.

Paranoid delusions were associated with being female and older in this sample, but there is no evidence from prior studies that this is the case generally. ${ }^{2,7,14}$ Hallucinations tended to occur in patients who were younger or who had a younger age of onset, but the rarity of hallucinations in this sample diminishes the importance of this finding. Interestingly, the greater the number of years of education the less likely it was that patients manifested delusions. Just as patients with higher baseline intellectual functioning may be able to compensate and conceal their cognitive deficits in the early stages of $A D$, so also they may be more adept at concealing suspicions or other psychotic features that may have developed.

In summary, the CUSPAD is a new screening instrument that uses explicit standardized questions for each item that render it suitable for use by trained lay interviewers. Interrater reliability and divergent validity have been established. The findings, particularly those pertaining to psychotic features, indicate the value of using standardized definitional criteria based on explicit questions to evaluate psychotic phenomenology. Clinical research evaluation based on new methodology of this type may prove to be of value in cross-sectional and longitudinal studies of psychopathology in AD.
This work was supported by federal grants AG07370, AG07232 AG08702, and MH44176, and the Charles S. Robertson Memorial Gift for research in Alzheimer's disease.

\section{References}

1. Seltzer B, Sherwin I. A comparison of clinical features in early- and late-onset primary degenerative dementia. Arch Neurol. 1983;40:143146.

2. Reisberg B, Borenstein J, Salob SP, Ferris SH, Franssen E, Georgotas A. Behavioral symptoms in Alzheimer's disease: phenomenology and treatment. / Clin Psychiatry. 1987;48(suppl 5):9-15.

3. Mace NL, Rabins PV. The 36-Hour Day. Baltimore, Md: Johns Hopkins University Press; 1981.

4. Deimling GT, Bass DM. Symptoms of mental impairment among elderly adults and their effects on family caregivers. J Gerontol. 1986; $41: 778-784$.

5. Terry RD, Katzman R. Senile dementia of the Alzheimer type. Ann Neurol. 1983;14:497-506.

6. Steele C, Rovner B, Chase GA, Folstein M. Psychiatric symptoms and nursing home placement of patients with Alzheimer's disease. Am I Psychiatry. 1990;147:1049-1051.

7. Rubin E, Drevets W, Burke W]. The nature of psychotic symptoms in senile dementia of the Alzheimer type. J Geriatr Psychiatry Neurol. $1988 ; 1: 16-20$.

8. American Psychiatric Association. Diagnostic and Statistical Man ual of Mental Disorders, Revised Third Edition. Washington, DC: American Psychiatric Association; 1987.

9. Devanand DP, Sackeim HA, Mayeux R. Psychosis, behavioral disturbance, and the use of neuroleptics in dementia. Compr Psychiatry. 1988;29:387-401.

10. McKhann G, Drachman D, Folstein M. Clinical diagnosis of Alzheimer's disease: report of the NINCDS-ADRDA Work Group under the auspices of Department of Health and Human Services Task Force on Alzheimer's Disease. Neurology. 1984;34:939-944.

11. Hughes CP, Berg L, Danziger WL, Cohen LA, Marin RL. A new clinical scale for the staging of dementia. Br J Psychiatry. 1982;140:556572.

12. Mayeux R, Stern Y, Rosen J, Leventhal J. Depression, intellectual impairment and Parkinson's disease. Neurology. 1981;31:645-650.

13. Blessed $\mathrm{C}$, Tomlinson BE, Roth $M$. The association between quantitative measures of dementia and of senile changes in the the cerebral grey matter of elderly subjects. Br J Psychiatry. 1968;225:797-811.

14. Burns A, Jacoby R, Levy R. Psychiatric phenomena in Alzheimer's disease, I: disorders of thought content. Br J Psychiatry. 1990;157:72-76.

15. Bucht $G$, Adolfsson $R$. The comprehensive psychopathological rating scale in patients with dementia of Alzheimer type and multiinfarct dementia. Acta Psychiatr Scand. 1983;68:263-270.

16. Teri L, Larson EB, Reifler BV. Behavioral disturbance in dementia of the Alzheimer's type. I Am Geriatr Soc. 1988;36:1-6.

17. Rubin EH, Kinscherf DA. Psychopathology of very mild dementia of the Alzheimer type. Am / Psychiatry. 1989;146:1017-1021.

18. Burns A, Jacoby R, Levy R. Psychiatric phenomena in Alzheimer's disease, I: disorders of perception. Br / Psychiatry. 1990;157:76-81.

19. Mayeux R, Stern $Y$, Spanton S. Heterogeneity in dementia of the Alzheimer type: evidence of subgroups. Neurology. 1985;35:453-461.

20. Sim M, Sussman I. Alzheimer's disease: its natural history and differential diagnosis. I Nerv Ment Dis. 1962;135:489-499.

21. Knesevich JW, Martin RL, Berg L, Danziger W. Preliminary report on affective symptoms in the early stages of senile dementia of the Alzheimer type. Am / Psychiatry. 1983:140:233-245.

22. Rovner BW, Broadhead I, Spencer M, Carson K, Folstein MF. Depression and Alzheimer's disease. Am J Psychiatry. 1989;146:350-353.

23. Fischer $P$, Simanyi $M$, Danielczyk $W$. Depression in dementia of the Alzheimer type and in multi-infarct dementia. Am / Psychiatry. 1990; 147:1484-1487.

24. Reifler BV, Larson E, Hanley R. Coexistence of cognitive impairment and depression in geriatric outpatients. Am / Psychiatry. 1982;139:623-626.

25. Mackenzie TB, Robiner WN, Knopman DS. Differences between patients and family assessments of depression in Alzheimer's disease. Am I Psychiatry. 1989;146:1174-1178.

26. Burns A, Jacoby R, Levy R. Psychiatric phenomena in Alzheimer's disease, I: disorders of mood. Br J Psychiatry. 1990;157:81-86.

27. Burke WJ, Rubin EH, Morris JC. Symptoms of depression in dementia of the Alzheimer type. Alzheimer Dis Assoc Disord. 1988;2:356362 .

28. Reifler BV, Teri $L$, Raskind $M$, et al. Double-blind trial of imipramine in Alzheimer's disease patients with and without depression. Am / Psychiatry. 1989; 146:45-49.

29. Jenike MA. Monoamine oxidase inhibitors as treatment for depressed patients with primary degenerative dementia (Alzheimer's disease). Am J Psychiatry. 1985;142:763-764.

30. Greenwald BS, Kramer-Ginsberg E, Marin DB, et al. Dementia with coexistent major depression. Am I Psychiatry. 1989;146:1472-1478. 